\title{
10 Characterization Techniques for Planar Optical Microresonators
}

\author{
René M. de Ridder, Wico C.L. Hopman", and Edwin J. Klein \\ University of Twente, MESA+ Institute for Nanotechnology, Enschede, the Netherlands \\ "now at: Sensata Technologies, Almelo, the Netherlands
}

\begin{abstract}
Optical microresonators that are coupled to optical waveguides often behave quite similar to Fabry-Perot resonators. After summarising key properties of such resonators, three characterization methods will be discussed. The first involves the analysis of transmission and reflection spectra, from which important parameters like (waveguide) loss and coupling or reflection coefficients can be extracted. The second method is called transmission-based scanning near-field optical microscopy (T-SNOM) which allows to map out the intensity distribution inside a high-Q resonator with subwavelength resolution. For such resonators conventional SNOM suffers from inaccuracies introduced by the disturbing effect of the presence of the probe on the field distribution. T-SNOM avoids this problem by exploiting this disturbing effect. The third method is most applicable to large-size (many wavelengths across) resonators. It involves simultaneous analysis of scattered light and transmission/reflection spectra in order to relate spectral features to large-scale field distributions inside the resonator. Together, these techniques form a convenient toolbox for characterizing many different planar optical microresonators.
\end{abstract}

Keywords: optical microresonators; optical measurements; SNOM; NSOM; AFM; scattered-light analysis.

\subsection{Introduction}

Optical resonators have many applications, such as wavelength filtering, add-drop multiplexing of several wavelength channels, lasers, and field enhancement [1-4]. Recent developments in integrated optics towards high-refractive index contrast technology (e.g. silicon photonics [5]) and related fields such as photonic crystals have opened the road towards a strong miniaturisation of optical circuits. In particular microring resonators [e.g. 1, 2, 5, 6] and cavities in photonic crystals [e.g. 3-5, 710] have been intensively investigated. Integrated optical circuits with high functionality have been conceived $[2,11]$, based on arrays of microresonators. Conventionally, optical resonators are characterized by performing spectral transmission and/or reflection measurements. In these methods, the input-output relationships are analysed, and through device models, some data on internal parameters can be inferred. These methods will provide essential information on the functionality and 
performance of the device in a system. For relatively simple resonator structures consisting of a few discrete elements, such as basic Fabry-Perot resonators, these measurements are fully adequate. However, for more complicated structures it will often be highly desirable to "look" inside the structure. This is especially the case for nanostructured devices where small disturbances may strongly affect their optical performance. Being able to map out the optical field distribution inside the structure can be extremely helpful in diagnosing possible fabrication errors, and for optimising the design, for example in order to maximise the quality factor $Q$.

In this chapter we will show how some internal parameters can be extracted through analysis of spectral transfer functions. After that we will concentrate on two different methods for looking inside the box. One method -that can provide subwavelength resolution- is most applicable to rather small structures (up to about 50 $\times 50 \mu \mathrm{m}^{2}$ ) such as cavities in photonic crystals, whereas the second method finds its main application in analysing extended structures such as gratings up to several $\mathrm{mm}$ in length. We will start with an introductory section on Fabry-Perot-type resonators in order to briefly review their properties and key parameters that may also apply to more complicated structures.

\subsection{Fabry-Perot-Type Optical Resonators}

One of the simplest types of resonator is the Fabry-Perot interferometer or etalon. As shown in Fig. 10-1, it consists of two parallel semitransparent mirrors having power reflectivities $R_{1}$ and $R_{2}$, separated by a given distance $L$. The space between the mirrors is referred to as the cavity. We will restrict the treatment here to the case of plane waves at normal incidence to the mirrors. Many practical resonators that have a more complicated structure still behave quite similar to this simple Fabry-Perot interferometer, e.g., an optical waveguide having two discontinuities that act as mirrors (in the simplest Fabry-Perot solid state laser, these discontinuities are the end facets showing Fresnel reflection, Fig. 10-2a), or a ring resonator that is coupled to straight waveguides through the evanescent field (Fig. 10-2b). In that case, assuming lossless devices, the power coupling coefficients $\kappa_{1}$ and $\kappa_{2}$ of the directional couplers correspond to the power transmission coefficients $T_{i}=1-R_{i}$ of a Fabry-Perot. More generally, any cavity that is coupled to the outside world through two ports can at some level of abstraction be described as a Fabry-Perot resonator, for example a cavity in a photonic crystal (Fig. 10-2c), or -maybe less obvious- a finite-length grating (Fig. 10-2d).

Fig. 10-1 Schematic of Fabry-Perot resonator.

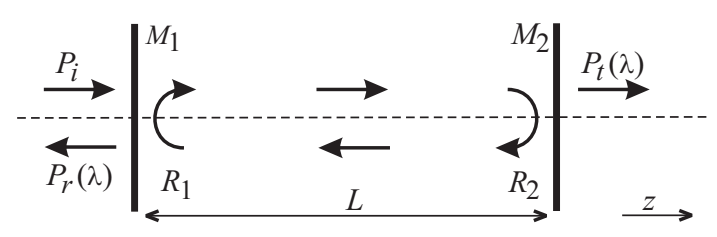




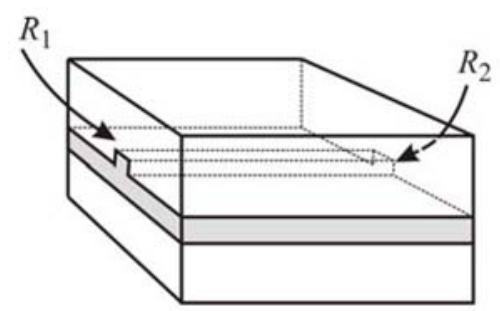

a)

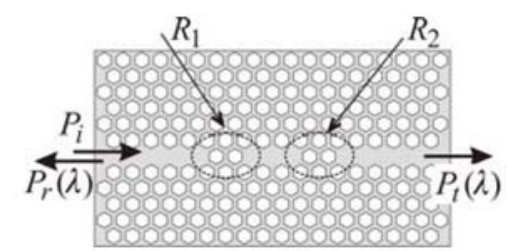

c)

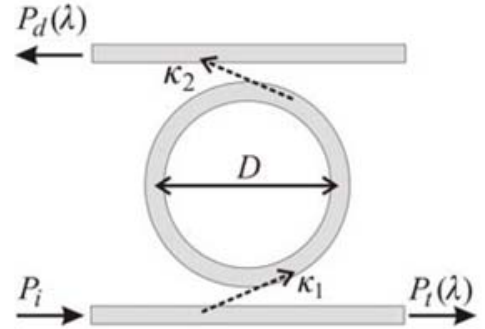

b)

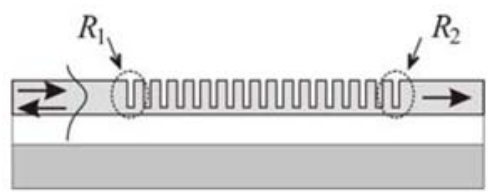

d)

Fig. 10-2 Examples of Fabry-Perot-like resonators: a) waveguide with reflecting facets; b) ring resonator; c) cavity in a photonic crystal; d) finite-length waveguide grating.

\subsubsection{Resonance Condition; Free Spectral Range (FSR)}

Resonance in a Fabry-Perot interferometer occurs if a wave after making a full roundtrip through the cavity (i.e. travelling twice the cavity length and reflecting once at each mirror) arrives in phase with itself. In a simple free-space Fabry- Perot, the phase shift at reflection is often neglected, and the roundtrip phase shift is determined by the optical path length $n L$, where $n$ is the refractive index inside the cavity. The resonance condition is given by

$$
\varphi=2 \beta L=m 2 \pi
$$

where $\varphi$ is the roundtrip phase shift, the resonance order $m$ is a positive integer, and $\beta$ is the propagation constant of the wave inside the cavity:

$$
\beta=\frac{\omega}{v_{p}}=\frac{\omega n}{c}=\frac{2 \pi}{\lambda} n
$$

with angular frequency $\omega$, phase velocity $v_{p}$, the speed of light in vacuum $c$, and the wavelength in vacuum $\lambda$. In waveguiding structures, the refractive index $n$ should be replaced by the effective refractive index $n_{\text {eff }}$.

Conditions (10.1) and (10.2) imply the occurrence of multiple resonance frequencies $\omega_{m}$ :

$$
\omega_{m}=m \pi c /(n L)
$$

The frequency (or wavelength) difference between two consecutive resonances is called the free spectral range (FSR). From (10.3) it seems to follow that the FSR is 
given by $\Delta \omega_{\mathrm{FSR}}=\pi c /(n L)$. However, this is valid only for nondispersive media ( $n$ is constant); with dispersive media we should be a bit more careful. From (10.1) we find for consecutive resonances:

$$
\Delta \varphi=2 \Delta \beta L=2 \pi .
$$

If dispersion is not too strong, and $\Delta \omega_{\mathrm{FSR}}$ is much smaller than the operating angular frequency, a linear approximation of dispersion is appropriate:

$$
\Delta \beta=\frac{\partial \beta}{\partial \omega} \Delta \omega,
$$

which, using (10.4) and the definitions of group velocity $v_{g}=\partial \omega / \partial \beta$ and group index $n_{g}=c / v_{g}$ leads to a more accurate expression for the FSR,

$$
\Delta \omega_{\mathrm{FSR}}=\pi c /\left(L n_{g}\right) .
$$

In terms of wavelength the FSR is expressed as

$$
\Delta \lambda_{\mathrm{FSR}}=\frac{1}{2} \lambda_{0}^{2} /\left(L n_{g}\right),
$$

where $\lambda_{0}$ is the wavelength halfway between two considered resonances. It is clear that smaller cavities show larger FSR.

In cases of strong dispersion and large FSR, the linear approximation of dispersion as used in (10.5) turns out to be not sufficiently accurate [12]. Analytic models are often lacking for the structures of interest, such as strong gratings and photonic crystal cavities. In such cases the dispersion curves $\beta(\omega)$ should be calculated numerically in order to verify the validity of (10.5) for the frequency range of interest. Another issue is the phase shift upon reflection. For simple cases such as Fresnel reflection at normal incidence at the interface between two dielectrics, the magnitude and phase of the reflection coefficient are well known. However, the situation is much less trivial if periodic structures are involved and evanescent waves occur that can be associated with a certain penetration depth into the reflecting medium. This leads to a frequency-dependent phase shift upon reflection, or, equivalently, a frequency-dependent effective cavity length. Such situations can be analysed using approximative coupled-mode models [13], or with "brute force" simulations, using e.g. finite-difference time-domain (FDTD) code.

\subsubsection{Transfer Function}

The transfer function of the simple Fabry-Perot can be calculated by considering the device as a feedback system for the electric field of the optical waves. The mirrors are characterized by their amplitude transmission and reflection coefficients $t$ and $r$ (transmitted field $E_{t}=t E_{\text {in }}$; reflected field $E_{r}=r E_{\text {in }}$ ); losses in the cavity are taken into account by assuming a complex propagation constant $\alpha+\mathrm{j} \beta$ where $\alpha$ is the attenuation constant and $\mathrm{j}$ is the imaginary unit, so that a travelling wave in the cavity can be represented as a phasor $E=E_{0} \mathrm{e}^{-\alpha z} \mathrm{e}^{-\mathrm{j} \beta z}$. 


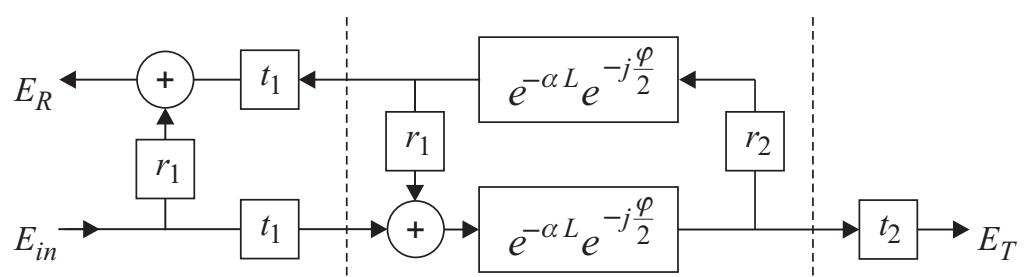

Fig. 10-3 Fabry-Perot cavity represented as a system with feedback. The block structure between the vertical dashed lines represents the process inside the cavity.

If we would not consider any back-reflections, the transmitted field through a cavity with length $L$ would be $E_{T}=E_{\text {in }} t_{1} t_{2} \mathrm{e}^{-\alpha L} \mathrm{e}^{-\mathrm{j} \varphi / 2}$ where we used (10.1). Taking into account the back-reflections results in a feedback scheme as illustrated by Fig. 10-3.

The field transmission of this system can be calculated to be

$$
E_{T}=E_{\text {in }} \frac{t_{1} t_{2} \mathrm{e}^{-\alpha L} \mathrm{e}^{-j \frac{\varphi}{2}}}{1-r_{1} r_{2} \mathrm{e}^{-2 \alpha L} \mathrm{e}^{-j \varphi}} .
$$

From this, the intensity transfer function is calculated as

$$
T_{\mathrm{FP}} \equiv I_{T} / I_{\mathrm{in}}=\left|E_{T} / E_{\mathrm{in}}\right|^{2} .
$$

Substitution of (10.8) into (10.9) gives:

$$
T_{\mathrm{FP}}=\frac{T_{0}}{1+B \sin ^{2}(\varphi / 2)},
$$

where $T_{0}$ and $B$ are constants determined by mirror reflectivities and cavity losses,

$$
\begin{gathered}
T_{0} \equiv \frac{\mathrm{e}^{-2 \alpha L}\left(1-R_{1}\right)\left(1-R_{2}\right)}{\left(1-\sqrt{R_{1} R_{2}} \mathrm{e}^{-2 \alpha L}\right)^{2}}, \\
B \equiv \frac{4 \sqrt{R_{1} R_{2}} \mathrm{e}^{-2 \alpha L}}{\left(1-\sqrt{R_{1} R_{2}} \mathrm{e}^{-2 \alpha L}\right)^{2}}, \\
R_{i}=\left|r_{i}\right|^{2} .
\end{gathered}
$$

Similarly the field reflection of this system can be calculated to be

$$
E_{R}=E_{\text {in }} \frac{r_{1}-r_{2} e^{-2 \alpha L} e^{-j \varphi}}{1-r_{1} r_{2} e^{-2 \alpha L} e^{-j \varphi}} .
$$

From this, the intensity reflection function is calculated as

$$
R_{\mathrm{FP}} \equiv I_{R} / I_{\text {in }}=\left|E_{R} / E_{\text {in }}\right|^{2} .
$$




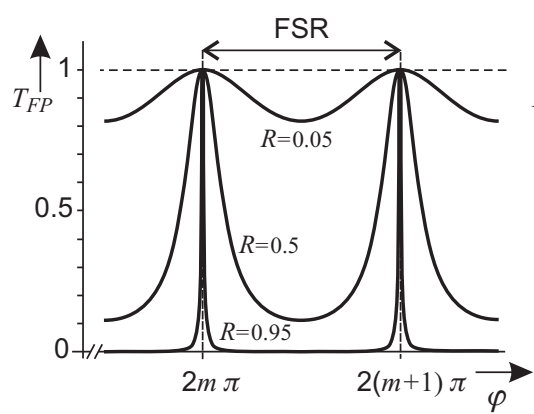

a)

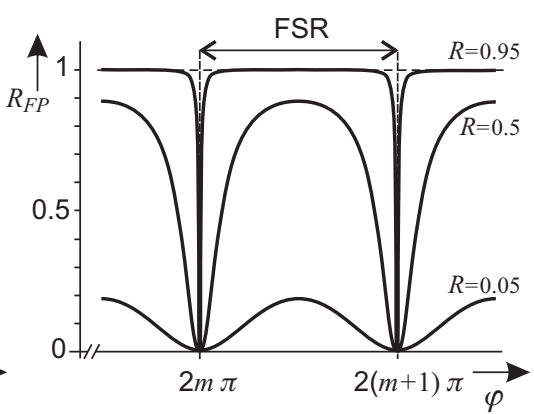

b)

Fig. 10-4 a) Transmission and b) reflection spectra of lossless Fabry-Perot resonator with equal mirrors for 3 different reflectance values.

Substitution of (10.14) into (10.15) gives:

$$
R_{\mathrm{FP}}=1-\frac{C}{1+B \sin ^{2}(\varphi / 2)},
$$

where $C$ is given by:

$$
C=\frac{\left(1-R_{1}\right)\left(1-R_{2} e^{-4 \alpha L}\right)}{\left(1-\sqrt{R_{1} R_{2}} e^{-2 \alpha L}\right)^{2}} .
$$

For the case of a lossless cavity $(\alpha=0)$, and identical mirrors $\left(R_{1}=R_{2}=R\right)$, Fig. 104 shows the transfer and reflection spectra $T_{\mathrm{FP}}(\varphi)$ and $R_{\mathrm{FP}}(\varphi)$ with different values of $R$ as parameter. It shows that high reflectivity is needed for obtaining narrow transmission peaks with deep minima in between.

\subsubsection{Width of a Transmission Peak (FWHM)}

The width of a resonance peak is usually given as the full width at half maximum (FWHM), i.e. the difference $\Delta \omega_{\mathrm{FWHM}}$ between two frequencies at both sides of the peak maximum, where the transmission is half that at the resonance frequency $\omega_{m}$. At frequencies $\omega_{m} \pm 1 / 2 \Delta \omega_{\mathrm{FWHM}},(10.10)$ should evaluate to $T_{0} / 2$, or

$$
\sin ^{2}(\varphi / 2)=1 / B
$$

Combining (10.1), (10.2), and (10.3) we rewrite $\varphi$ as:

$$
\varphi=m 2 \pi \frac{\omega}{\omega_{m}} \frac{n(\omega)}{n\left(\omega_{m}\right)}
$$

where we wrote the possibly dispersive refractive index explicitly as a function of frequency. Using (10.19) in (10.18) and substituting $\omega_{m} \pm 1 / 2 \Delta \omega_{\mathrm{FWHM}}$ for $\omega$, we get:

$$
\sin ^{2}\left\{m \pi \frac{\omega_{m} \pm \frac{1}{2} \Delta \omega_{\mathrm{FWHM}}}{\omega_{m}} \frac{n(\omega)}{n\left(\omega_{m}\right)}\right\}=\frac{1}{B} .
$$


In (10.20) we need to know the refractive index at three frequencies that are rather close together in high-quality resonators. Therefore, the error will be small (and evaluation of (10.20) much more convenient) if we neglect dispersion and set

$$
n\left(\omega_{m}-\frac{1}{2} \Delta \omega_{\text {FWHM }}\right)=n\left(\omega_{m}+\frac{1}{2} \Delta \omega_{\text {FWHM }}\right)=n\left(\omega_{m}\right),
$$

so that (10.20) simplifies to

$$
\sin ^{2}\left\{m \pi \pm \frac{1}{2} m \pi\left(\Delta \omega_{\text {FWHM }} / \omega_{m}\right)\right\}=1 / B .
$$

If the cavity losses are sufficiently low, then $1 / B$ will be a small number, and only a small error will be made if we replace the sine function by its argument (e.g., for $R>$ 0.9 and $\alpha L<0.05, B \geq 90, \sin (.) \leq$.0.01 , and the error made in the value of the argument will be below $0.2 \%$ ). Due to the symmetry and periodicity of $\operatorname{the}^{2} \sin ^{2}()$ function, the $m \pi$ term and the \pm sign can be safely omitted, leading to:

$$
\Delta \omega_{\mathrm{FWHM}}=\omega_{m} \frac{2}{m \pi \sqrt{B}} .
$$

\subsubsection{Quality Factor $Q$}

A quantity of great practical importance is the quality factor $Q$, which can be defined in a number of equivalent ways. An operational definition that allows direct measurement of $Q$ is the relative peak width, which can also be directly derived from (10.23):

$$
Q \equiv \omega_{m} / \Delta \omega_{\mathrm{FWHM}}=\frac{1}{2} m \pi \sqrt{B} .
$$

For frequencies near resonance, (10.10) can be written as a Lorentzian function, in terms of the resonance frequency $\omega_{m}$ and $Q$ only. Near resonance, we write:

$$
\omega=\omega_{m}+\Delta \omega
$$

with $\Delta \omega<<\omega_{m}$. This allows us to again neglect dispersion, so that the argument in the sine function in (10.10) becomes similar to that in (10.22) with $\Delta \omega_{\text {FWHM }}$ replaced by $\omega_{m}-\omega$ :

$$
\sin ^{2}(\varphi / 2)=\sin ^{2}\left(m \pi+m \pi\left(\omega_{m}-\omega\right) / \omega_{m}\right) \cong\left(m \pi\left(\omega_{m}-\omega\right) / \omega_{m}\right)^{2} .
$$

Inserting (10.26) into (10.10), and using (10.24), results in an expression, valid only for a narrow frequency range centred on a resonance peak, showing the Lorentzian shape of such peaks:

$$
T(\omega) \cong \frac{T_{0}}{1+B\left(\frac{m \pi}{\omega_{m}}\right)^{2}\left(\omega_{m}-\omega\right)^{2}}=T_{0} \frac{1}{1+\left(\frac{2 Q}{\omega_{m}}\right)^{2}\left(\omega_{m}-\omega\right)^{2}} .
$$

With (10.23), an equivalent expression can be found in terms of $\omega_{m}$ and $\Delta \omega_{\mathrm{FWHM}}$. 


\subsubsection{Field Distribution}

The operation of a Fabry-Perot resonator was introduced with Fig. 10-1, showing forward and backward propagating waves in the cavity. These travelling waves interfere to form standing waves, as shown schematically in Fig. 10-5. The order of such resonant modes is given by the integer $m$ that occurs in (10.1).

Low-order resonances are usually easily identified by their frequencies, which are relatively wide apart. For higher-order modes, the relative frequency difference $\left(\omega_{m+1}-\omega_{m}\right) / \omega_{m}$ becomes smaller, making it more difficult to identify a particular resonant mode.

At resonance, the field strength inside the cavity may become much larger than the input field. Looking at the schematic of Fig. 10-3, it can be seen that the amplitude $E_{t}$ of the output wave is a factor $t$ smaller than $E_{\text {right }}$ of the right-travelling wave inside the cavity. On the other hand, we know that for a lossless cavity at resonance $E_{\text {out }}=E_{\text {in }}$. The amplitude $E_{\text {left }}$ of the left-travelling wave inside the cavity is a factor $r$ smaller than $E_{\text {right. }}$.

Intensity maxima in the cavity arise where right- and left-travelling waves are in phase so that they add up to $E_{\text {max }}=E_{\text {left }}+E_{\text {right }}=E_{\text {right }}(1+r)=E_{\text {in }}(1+r) / t$. Using $t^{2}=$ $T=1-R$, and $r^{2}=R$, the intensity enhancement is calculated as

$$
I_{\text {max,cavity }} / I_{\text {in }}=\left(E_{\text {max,cavity }} / E_{\text {in }}\right)^{2}=(1+\sqrt{R})^{2} /(1-R),
$$

which becomes quite large if $R$ approaches 1 . This phenomenon is often used for local enhancement of optical effects, in particular nonlinear optics. The enhancement is limited by optical loss. In the case of planar optics not only propagation loss due to absorption or scattering should be considered but also out-of-plane diffraction, especially with periodic structures such as gratings and photonic crystals.

Fig. 10-5 Schematic picture of the lowest 3 longitudinal resonant modes in a FabryPerot resonator.
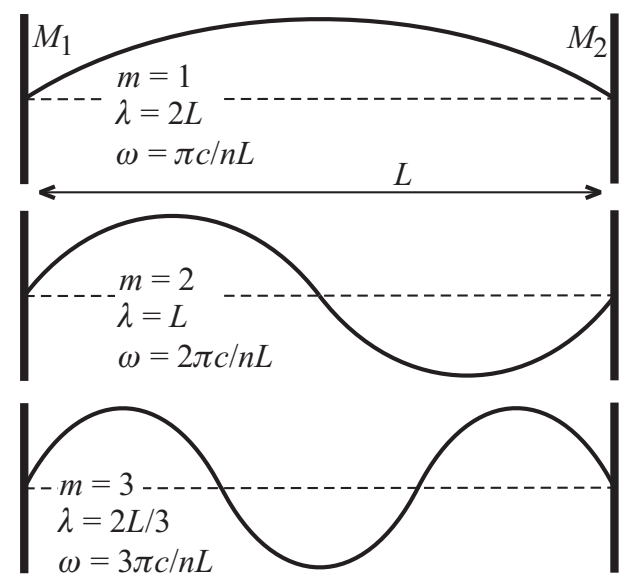


\subsubsection{Higher-Order Transversal Modes; Degeneracy}

In guided-wave optics the light is confined in transversal directions (perpendicular to the direction of propagation) by dielectric waveguides. If the waveguide inside the Fabry-Perot cavity supports only a single guided mode (Fig. 10-6a), the analysis given above is valid if the refractive index is replaced by the effective index of the waveguide mode.

$$
n_{\text {eff }}=\beta \lambda /(2 \pi),
$$

where $\beta$ is the modal propagation constant and $\lambda$ is the wavelength in vacuum.

If the waveguide supports multiple modes, things get a bit more complicated. In the example of Fig. 10-6b, two modes are considered, having slightly different propagation constants $\beta_{0}$ and $\beta_{1}$, hence slightly different effective indices $n_{\text {eff }, 0}$ and $n_{\text {eff, }, 1}$. The resonance conditions (10.1) for these modes will therefore be somewhat different, so that in general these modes will be in resonance at different frequencies.

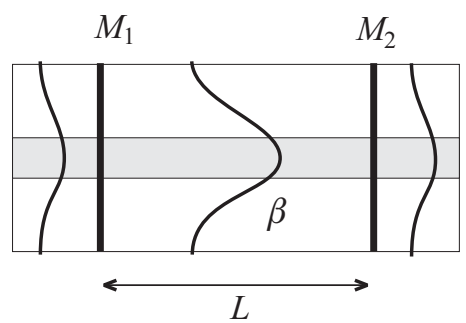

a)

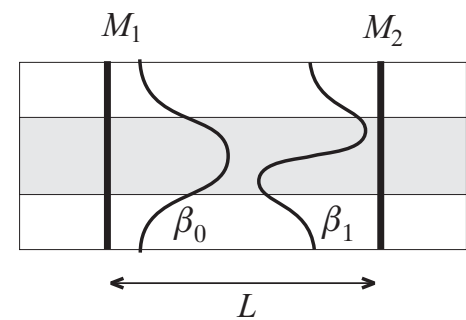

b)

Fig. 10-6 Waveguide-based Fabry-Perot resonator; the mirrors are represented symbolically as thick vertical lines. a) single-mode waveguide; b) bimodal waveguide.

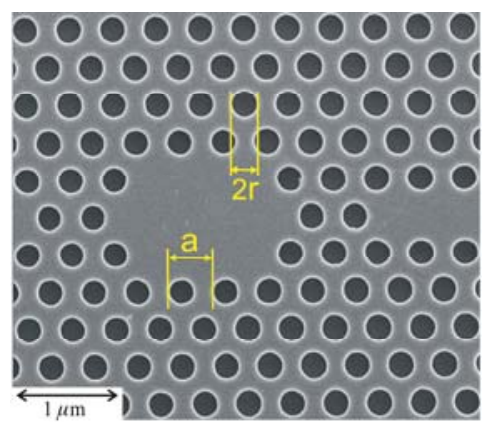

a)

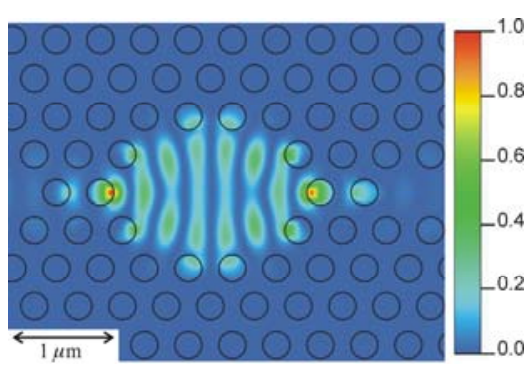

b)

Fig. 10-7 Fabry-Perot-like resonator in a photonic crystal slab. a) Scanning electron microscope (SEM) image of the device that was fabricated (at IMEC, Leuven, Belgium) in a silicon on insulator (SOI) technology [5]; silicon $(n=3.45)$ top layer thickness $220 \mathrm{~nm}$, lattice period $a=440 \mathrm{~nm}$, air hole radius $r=270 \mathrm{~nm}$. b) Intensity distribution of resonant mode at $\lambda=1550 \mathrm{~nm}$, calculated with a 2D-finite-difference time-domain (FDTD) method, using an effective index method for determining the silicon slab effective index $n_{\text {eff }}=2.9$. [14] 
However, at a given frequency one transversal mode may resonate at order $m_{0}$, whereas the other mode resonates at a different order $m_{1}$. This occurs if $n_{\text {eff }, 0} / m_{0}=$ $n_{\text {eff, }, 1} / m_{1}$.

In that case the standing wave pattern can be an arbitrary superposition of the two resonant modes, which are then said to be degenerate. In a more general case of nonrectangular two-dimensional resonators, the transversal (waveguide) and longitudinal (Fabry-Perot) modes cannot be determined independently, and it is a nontrivial task to calculate the composite modes. An example of such a resonator, in this case a cavity in a photonic crystal, schematically shown in Fig. 10-2c, can be seen in Fig. 10-7 together with a typical modal intensity distribution.

\subsection{Spectral Analysis}

Transmission and reflection spectra (or "drop" and "through" spectra in the case of ring resonators) represent the important basic filtering or add-drop multiplexing functionality of these devices. These spectral filter characteristics can usually be measured conveniently using either a white-light source in combination with a spectrometer, or a tuneable laser with a suitable photodetector.

\subsubsection{Determining Cavity Loss and Mirror Reflectivities}

An important parameter that is not always easy to measure directly is the loss of a mode in a waveguide. Often other origins of wave attenuation, such as fibre-chip coupling loss, and unknown cavity mirror reflectivities or coupling coefficients make propagation loss measurements difficult. Knowledge of propagation loss is important for the operation of resonators $(Q)$, and for almost all other applications.

The spectral response curves of a Fabry-Perot-type resonator provide sufficient information to calculate propagation loss $\alpha$ as well as the reflectivities $R_{i}$ (or coupling coefficients $\kappa_{i}$ in the case of ring resonators). The equations required to obtain the values of $\alpha$ and $R_{i}$ can be found from the expressions that give the on- and offresonance power ratios of the transmitted and reflected spectra.

The on/off resonance power ratio $M_{T}$ of the signal that is transmitted by the Fabry-Perot is defined as the ratio of the transferred power at resonance $(\varphi=m 2 \pi)$ to the transferred power at anti-resonance $(\varphi=m 2 \pi+\pi)$ :

$$
M_{T}=\frac{T_{\text {OnRes }}}{T_{\text {OffRes }}}=\left.\frac{\left.\frac{T_{0}}{1+B \sin ^{2}(\varphi / 2)}\right|^{\varphi=2 \pi}}{\frac{T_{0}}{1+B \sin ^{2}(\varphi / 2)}}\right|^{\varphi=\pi}=1+B=\left(\frac{1+\sqrt{R_{1} R_{2}} e^{-2 \alpha L}}{1-\sqrt{R_{1} R_{2}} e^{-2 \alpha L}}\right)^{2},
$$

which can be rewritten as:

$$
\sqrt{R_{1} R_{2}} e^{-2 \alpha L}=\frac{\sqrt{M_{T}}-1}{\sqrt{M_{T}}+1} .
$$


Similarly the on/off resonance reflected power ratio $M_{R}$ is defined as:

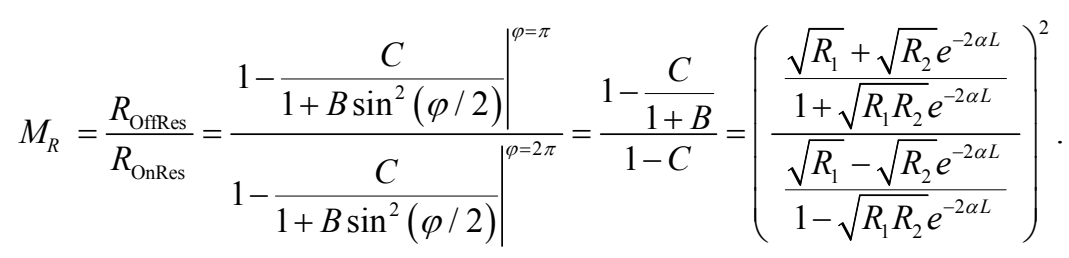

In order to obtain an explicit expression for $\alpha$, we first take the square root of the product of (10.30) and (10.32):

$$
\sqrt{M_{R} M_{T}}=\frac{\sqrt{R_{1}}+\sqrt{R_{2}} e^{-2 \alpha L}}{\sqrt{R_{1}}-\sqrt{R_{2}} e^{-2 \alpha L}},
$$

which can be rearranged to give:

$$
\frac{\sqrt{R_{2}}}{\sqrt{R_{1}}} e^{-2 \alpha L}=\frac{\sqrt{M_{R} M_{T}}-1}{\sqrt{M_{R} M_{T}}+1} .
$$

For Fabry-Perot cavities with $R_{1}=R_{2}=R$ this reduces to:

$$
e^{-2 \alpha L}=\frac{\sqrt{M_{R} M_{T}}-1}{\sqrt{M_{R} M_{T}}+1}
$$

which, combined with (10.31) can be used to find the values of $\alpha$ and $R$.

\subsection{Transmission-Based SNOM (T-SNOM)}

\subsubsection{Origin of the Method}

Scanning near-field optical microscopy (SNOM, NSOM) is a well-known method for probing optical field distributions with sub-wavelength resolution [15-17]. The basic principle is shown in Fig. 10-8. An optical fibre which is modified to have an extremely sharp tip (diameter in the order of $100 \mathrm{~nm}$ ) is brought in close proximity to the optical field to be probed. For obtaining the highest resolution, the tip is often clad with metal. At the very end of the tip a tiny hole is made with a diameter ranging from a few tens to a few hundreds of nm. It would typically be positioned in the evanescent field tail of guided waveguide modes. A small amount of light is scattered by the presence of the tip and collected by the fibre that guides the photons to a sensitive optical detector.

By scanning the tip across the surface of the device under analysis, and recording the amount of light captured by the fibre at each position, an image can be constructed that may represent the local optical intensity distribution. A problem with this method is that the probe may affect the optical field distribution. This is especially problematic when analysing microcavities, since the original structure will be significantly modified by the mere presence of the dielectric fibre material and its metal cladding. 
Fig. 10-8 Fibre tip of scanning near-field optical microscope (SNOM) interacting with the evanescent field of a resonant mode of a microcavity in a photonic crystal slab (crosssectional view).

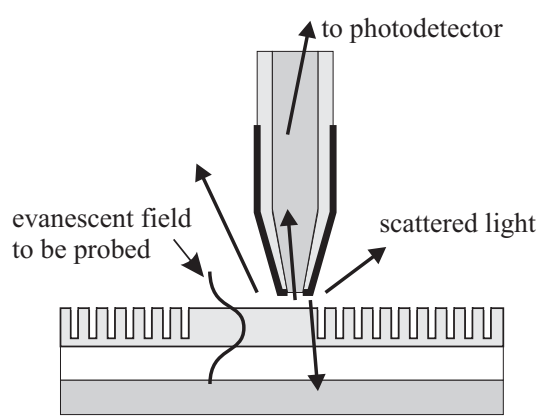

Also the light scattering process that is essential for SNOM operation may be an important loss mechanism which can strongly affect $Q$ of a resonant structure. Only a small fraction of the scattered light will be captured by the fibre and guided towards the detector, so that sensitivity and noise issues may easily arise.

In our work we originally intended to use SNOM measurements for characterizing the microresonators that we designed and fabricated. Because of the considerations mentioned above, we wanted to test the loading effect of a SNOM-like probe on a microresonator.

In order to check the effects of a probe, we measured the transmission through the Fabry-Perot-like resonator while scanning the probe over the device. For convenience we used an atomic force microscope (AFM) with a $\mathrm{Si}_{3} \mathrm{~N}_{4}$ or a $\mathrm{Si}$ tip with a radius in the order of $10 \mathrm{~nm}$, which is significantly smaller than a typical SNOM tip. Still, we observed a strong effect of the probe on the light transmission through the resonator. We decided to turn this drawback into an advantage, as shown below.

\subsubsection{Set-Up}

It should be expected that the strongest interaction of the nanoprobe with the optical field will occur at locations with highest field intensity.

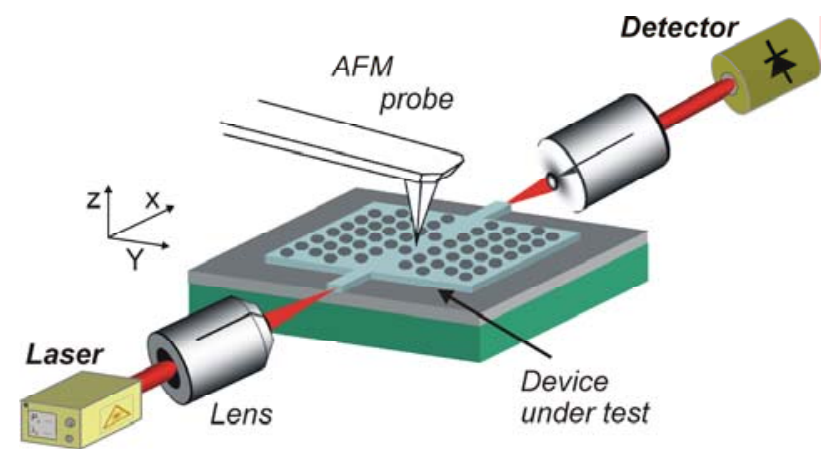

Fig. 10-9 Principle of transmission-based scanning near-field optical microscopy (T-SNOM) [14]. 
The effect of the probe may be both loss (due to scattering or waveguiding inside the probe), which mainly affects $Q$, and detuning of the cavity (the probe forms a "dielectric load" or becomes part of the cavity, thus changing its shape and effective index), bringing it into or out of resonance for a given frequency. If we measure the transmitted light through the cavity for each position of the probe, we can map out the locations of strong interaction on the surface of the resonator. The setup is shown schematically in Fig. 10-9. Since the image is obtained by scanning the optical near field with a probe while the signal is derived from the optical transmission through the device under test, we named this method transmission-based scanning near-field optical microscopy (T-SNOM).

\subsubsection{Image Construction - Contrast}

The method has two main advantages compared to conventional SNOM. The measurement is in fact a comparison between the undisturbed situation (no probe interacting with the optical field) and the disturbed situation, whereas in SNOM only photons are detected in the disturbed situation. A low optical SNOM signal may therefore be caused either by a small intensity in the undisturbed case, or it can be due to the disturbance by the probe itself that may cause the resonator to go out of resonance, which can only happen when the probe is at a location of high undisturbed intensity. With T-SNOM we actually exploit this disturbance, giving rise to less ambiguity in the result: a strong change of the optical signal will always be caused at a probe position with high undisturbed intensity. The second advantage is that the T-SNOM optical signal can be much stronger than that of SNOM. A conventional SNOM probe only detects the photons that are captured by the fibre. For obtaining high spatial resolution the opening in the tip should be small and the tunnelling efficiency of photons through this aperture becomes low. This may give rise to noise problems and, hence, long signal integration times. By contrast, a T-SNOM image is typically obtained with the undisturbed device in resonance, so that a large optical signal is transmitted to the detector. The probe interaction causes sharp dips in this transmission. This typically results in a better signal to noise ratio. A drawback of T-SNOM is that it works well only for resonant structures or other interferometric devices. The loss induced by the tiny probe on a large waveguide will be rather small, resulting in low contrast images. On the other hand, this is a situation where SNOM is generally reliable, so the two methods are mutually complementary. It should be mentioned that SNOM is a more versatile instrument since it does not depend on waveguiding structures and it can also be used in a mode that the fibre tip is used for locally illuminating a sample.

Using an AFM scanning head makes it possible to generate topographic data simultaneously with the optical data. Traditional SNOM provides similar facilities. The result of a T-SNOM measurement of the photonic crystal resonator shown in Fig. 10-7a is given in Fig. 10-10. The AFM (with a $10 \mathrm{~nm}$ radius $\mathrm{Si}_{3} \mathrm{~N}_{4}$ tip, shown in Fig. 10-11a) was operating in contact mode with closed-loop control. 


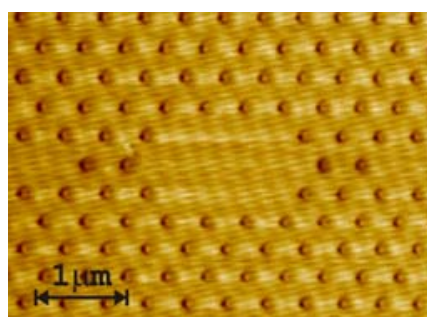

a)

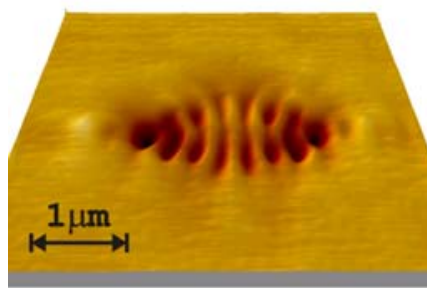

b)

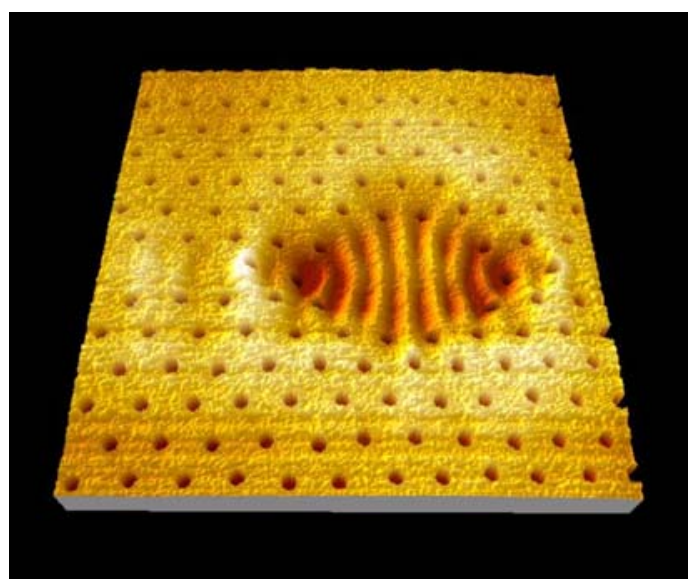

c)

Fig. 10-10 T-SNOM imaging of the photonic crystal resonator shown in Fig. 10-7a. a) Topography measured by AFM. b) Optical transmission through the resonator at the undisturbed resonance wavelength $\lambda_{r}=1539.25 \mathrm{~nm}$, as a function of AFM tip position; dark regions correspond to low light transmission, hence strong interaction of the AFM tip with the optical field. c) Composite image, combining data of a) and b), showing the location of the optical field distribution with respect to the resonator geometry. [14]

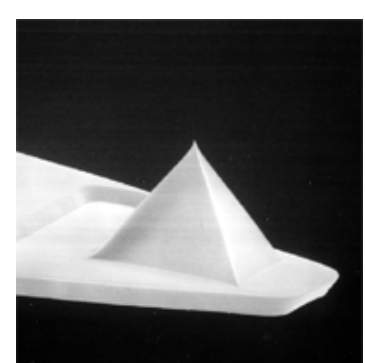

a)

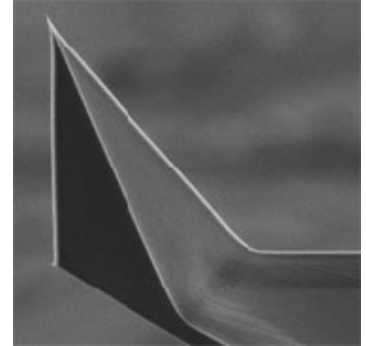

b)

Fig. 10-11 SEM images of AFM probes used. a) Silicon nitride with top angle $23^{\circ}$ and tip radius $10 \mathrm{~nm}$. b) Silicon with $17^{\circ}$ and tip radius $7.5 \mathrm{~nm}$.

In our experiments the scanning speed was limited by the response time of the photodetector. The image of Fig. 10-10, having a resolution of $256 \times 256$ pixels on a $20 \mathrm{~nm}$ grid, was taken in approximately $45 \mathrm{~s}$.

Using this small dielectric tip with a refractive index $(n=2.0)$ smaller than the silicon slab effective index $\left(n_{\text {eff }} \cong 2.9\right)$, the dominant effect of the probe on the resonator was detuning (shifting the resonant wavelength to larger values) rather than inducing scattering loss. This is illustrated by Fig. 10-12 showing a T-SNOM image of the same resonator, taken at a slightly larger wavelength of $1541.5 \mathrm{~nm}$, where the device is off resonance. The tuning action of the tip brings it on resonance again, now producing increased light transmission at locations of strong mechano-optical interaction. This resulted in an image with inverted contrast. 


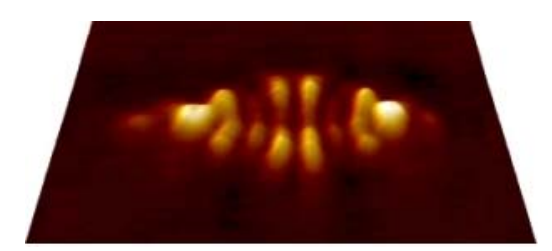

Fig. 10-12 T-SNOM image similar to Fig. 10-10, but wavelength, now $1541.5 \mathrm{~nm}$ (off resonance). Detuning by the AFM tip could bring it into resonance again, resulting in inverted image contrast. [14]

\subsubsection{Measurements of Nanomechano-Optical Interaction}

We measured the effect of small horizontal and vertical tip displacements on the tuning and on the quality factor of the resonator. Most of these experiments were

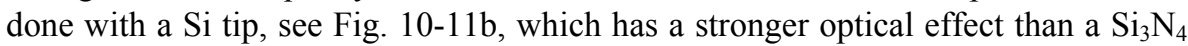
one, because of its higher refractive index $(n=3.45)$.

Transmission spectra for different tip positions in contact with the resonator surface are shown in Fig. 10-13a. Positioning the tip right at the hot spot (labelled A in the inset of the figure), almost completely kills the resonance. The dependence of the transmission on the distance of the tip from the hot spot is shown in Fig. 10-13b. For this structure the vertical dependence is especially strong: less than $100 \mathrm{~nm}$ displacement of the tiny tip results in more than $50 \%$ modulation of the transmission: this is a truly nanomechano-optical effect.

The tuning effects of the $\mathrm{Si}$ and $\mathrm{Si}_{3} \mathrm{~N}_{4}$ AFM tips have been compared in Fig. 1014. As expected, the $\mathrm{Si}$ tip has a much stronger effect than the $\mathrm{Si}_{3} \mathrm{~N}_{4}$ one, especially on the cavity loss. For tuning purposes the $\mathrm{Si}_{3} \mathrm{~N}_{4}$ tip is preferred.

\subsubsection{Modelling T-SNOM}

T-SNOM has been modelled in order to verify our intuitive understanding, and its operation has been simulated [18]. The purpose was to check whether the T-SNOM image does represent the standing wave pattern in a resonator with sufficient accuracy. A full and truly accurate model of T-SNOM should be three-dimensional. Because this is not practical with the currently available computing resources, the models were restricted to two dimensions. Two models have been used, a crosssectional view in a plane perpendicular to the photonic crystal slab ("side view"), and an effective-index-based "top view", see Fig. 10-15. 


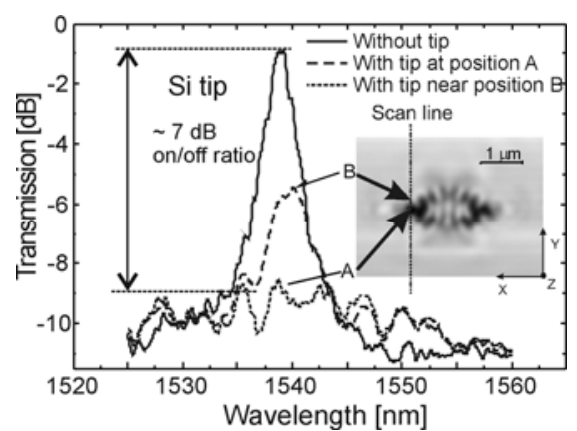

a)

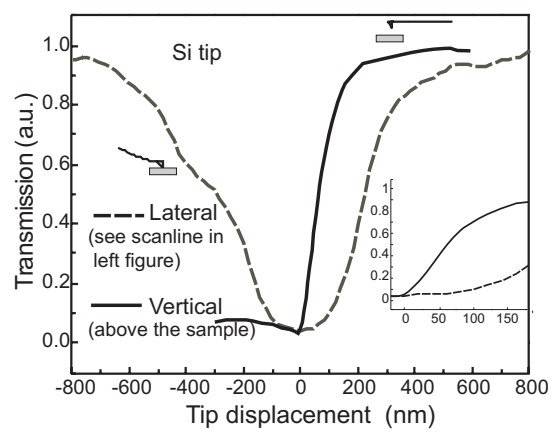

b)

Fig. 10-13 Nanomechano-optical interaction. a) Transmission spectra for the situation without probe; with a Si probe positioned at the antinode labelled A; and at a location B, as indicated in the inset. b) Transmission (at $\lambda=1539.25 \mathrm{~nm}$ ) versus Si tip displacement in the $z$ and $y$ direction (see the dotted "scan line" in the inset of the left figure). [14]

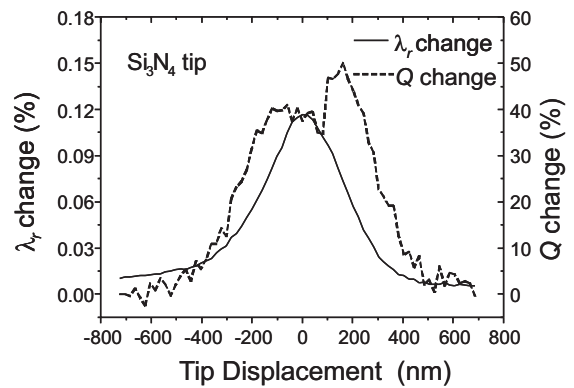

a)

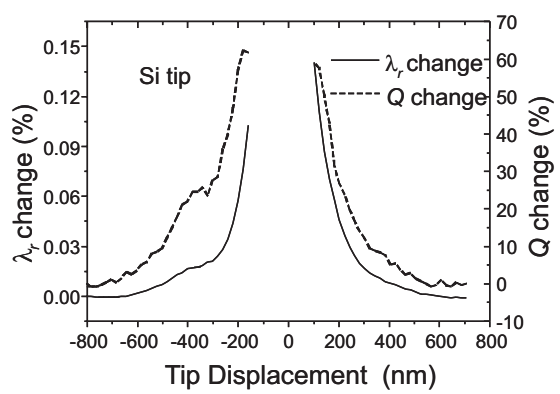

b)

Fig. 10-14 Comparison of different tips. Nanomechano-optical tuning of the resonance wavelength and $Q$ by lateral displacement with respect to hot spot $\mathrm{A}$, in contact along the scanline shown in the inset of Fig. 10-13a. a) $\mathrm{Si}_{3} \mathrm{~N}_{4}$ probe; b) Si probe. Close to the hot spot no data could be obtained because the transmission had dropped below the noise level. [14]

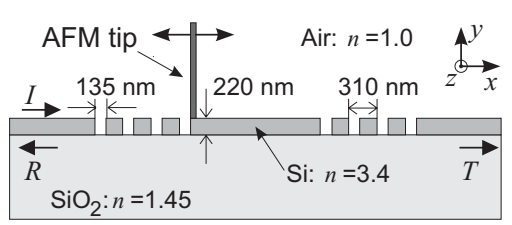

a)

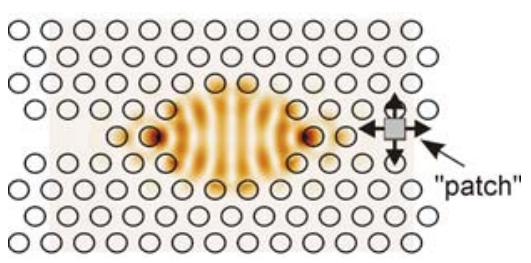

b)

Fig. 10-15 Two-dimensional T-SNOM models. a) Side view. The resonator is modelled as a slab waveguide cavity enclosed by two short Bragg gratings; the AFM-probe is modelled as a semiinfinite plane perpendicular to and in contact with the slab. b) Top view. The slab waveguide outside the photonic crystal holes is modelled by its effective index; the probe is represented by a small "patch" of material having an increased refractive index. 


\subsubsection{Side-View Model}

The side-view model is particularly suited for evaluation using an eigenmode propagation method, because of the limited number of different cross-sections, all with rectangular-shaped elements, making it possible to subdivide the region in a small number of horizontal layers and vertical slices. In this case the so-called quadrilateral eigenmode propagation (QUEP) [19] has been selected.

The model was first used to calculate the transmission, reflection and scattering spectra for several probe positions. The results for the undisturbed situation and the maximally disturbed case are shown in Fig. 10-16. As the next step, the model was recalculated at the undisturbed resonance wavelength, for many positions of the probe along the length of the resonator, thus simulating the scanning action of the tip. The resulting transmission versus tip position graph is shown in Fig. 10-17a.

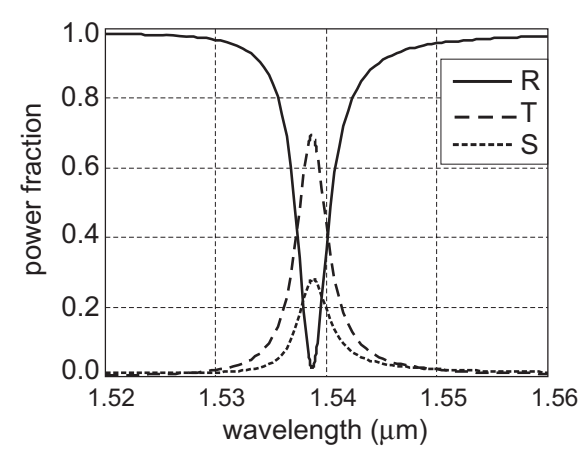

a)

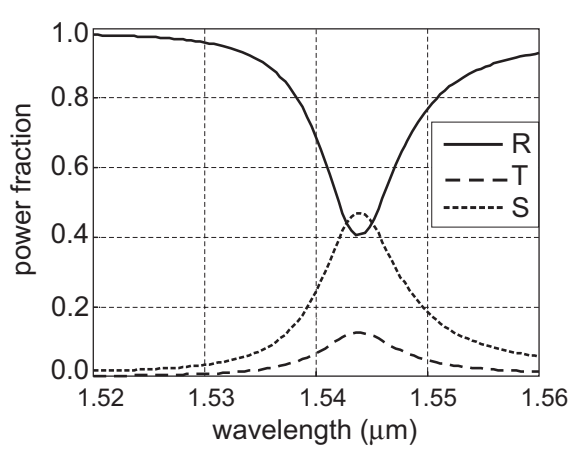

b)

Fig. 10-16 Calculated reflection $(R)$, transmission $(T)$ and scattered power $(S)$ spectra; $R+T+S=1$. a) Unperturbed resonator. b) Silicon probe (50 nm wide) at "hot spot" location. [18]

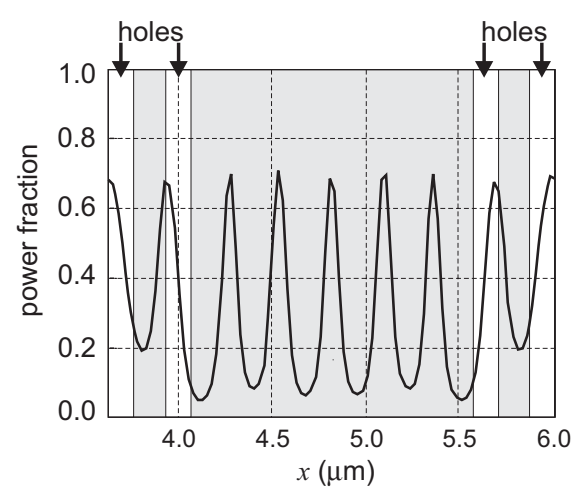

a)

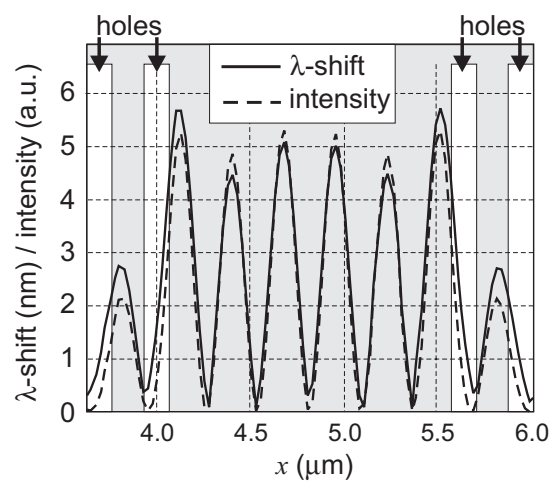

b)

Fig. 10-17 Simulation results. a) Transmission versus tip position. b) Solid curve: wavelength shift as calculated by combining the transmission graph (this figure, part a.) with the transmission spectrum of Fig. 10.16a. Dashed curve: calculated intensity distribution in the resonator. 
The changes in transmission in this graph have been translated into equivalent shifts of the resonance wavelength, using the previously measured spectra of Fig. 10-16. This equivalent wavelength shift has been plotted together with the calculated intensity distribution in Fig. 10-17b. The two graphs match quite well, indicating that indeed T-SNOM data can be used for determining field distributions inside this type of resonators.

Another calculation, illustrated in Fig. 10-18, simulates the effect of probe height variation. In contact $(y=0)$, the transmission is low (strong detuning), and as the tip is raised the transmission increases. It is interesting to see that at a distance of approximately $0.5 \mu \mathrm{m}$, there is a maximum transmission which is higher than in absence of the probe (large $y$ ). This is somewhat unexpected because the wavelength was selected for resonance without the probe. In fact, this simulation reproduces an effect that can also be seen in the measurement data of Fig. 10-10. At both the lefthand and the right-hand sides of the cavity there is a small region with a somewhat brighter colour than the background, signalling an increased transmission as well. The calculated field distributions without the tip and with the tip at the "critical height" of $0.5 \mu \mathrm{m}$ (Fig. 10-19), show that the presence of the tip at that location somewhat suppresses the radiation loss due to out-of-plane diffraction.

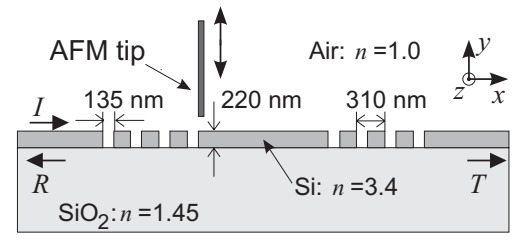

a)

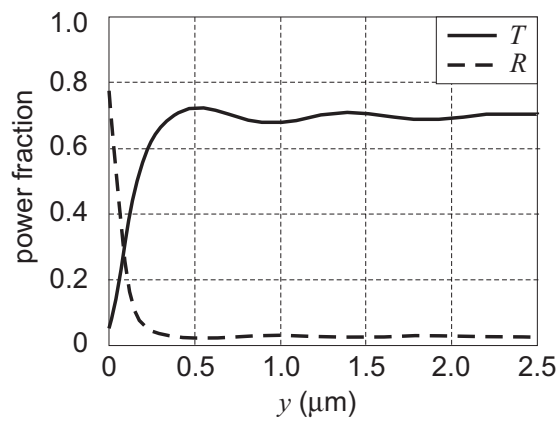

b)

Fig. 10-18 Simulation of probe height variation. a) Configuration: the z-position of the probe is at the hot spot; $y$ is varied. b) Calculated transmission and reflection versus probe height. [18]

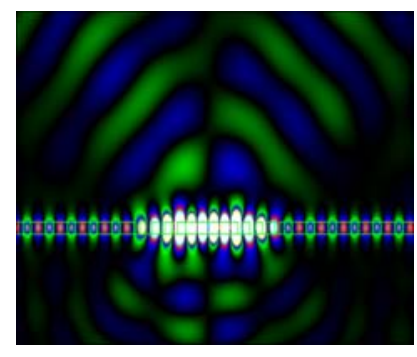

a)

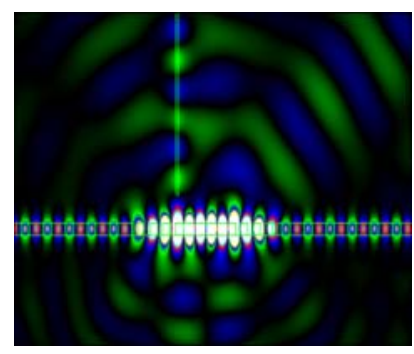

b)

Fig. 10-19 Calculated field distributions at the undisturbed resonance wavelength. a) Without probe. b) With probe at $0.5 \mu \mathrm{m}$ height above hot spot. [18] 
The space between the resonator surface and the probe tip acts as an additional cavity, which is in antiresonance at the critical height.

A similar model has also been used for modelling the dynamic behaviour of TSNOM when it is operated in so-called tapping mode (introducing a vertical vibration of the tip) [20]. The strongly nonlinear relationship between transmission and tip height requires a suitable weighing function in order to obtain the correct timeaveraged transmitted power. Tapping mode operation is beneficial for tip durability and for reducing the risk of producing artefacts by dragging along dust particles with the tip, but it reduces the lateral resolution.

\subsubsection{Top-View Model}

The top-view model, which is illustrated in Fig. 10-15b, is numerically somewhat more challenging than the side-view model because of the lack of straight interfaces. This makes it impractical to use an eigenmode propagation method, because the number of slices should be chosen very large in order to obtain a good approximation to the geometry of the problem. Because eigenmode calculations for all these slices are expensive, another method -the two-dimensional finite-difference timedomain (FDTD) method- usually considered as "brute force" comes out more favourably.

In this model, the vertical structure is accounted for by a local effective index. The silicon slab making up the "backbone" of the structure is represented by the effective index that it would have for a guided optical slab mode; the photonic crystal holes are approximated by an effective index of 1 (air), and the AFM probe is accounted for by simulating its dielectric loading effect through a locally increased effective index (the "patch" in Fig. 10-15b). The results, shown in Fig. 10-20, confirm that T-SNOM measurements in Fabry-Perot-like resonators provide a good approximation of the actual intensity distribution of the standing wave pattern inside the resonator.

In conclusion, T-SNOM is a useful extension of the available techniques for characterizing resonant optical structures. It provides a way to map out the intensity distribution inside a resonator, thus resolving the mode structure. It helps to identify

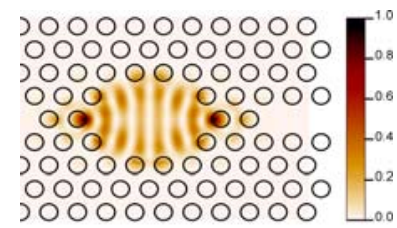

a)

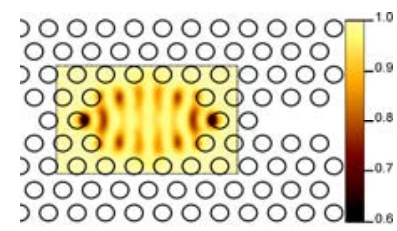

b)

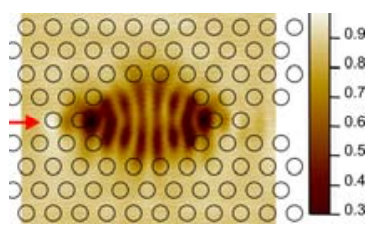

c)

Fig. 10-20 Top-view model, comparison to measurement. a) Calculated intensity distribution. b) Simulated T-SNOM response by recalculating the model many times for different "patch" locations in order to represent the T-SNOM probe scanning. c) Actual T-SNOM measurement data overlaid with structure data. [18] 
"hot spots" that are particularly useful for nanomechano-optical interaction that can be exploited for sensors and extremely compact and low power optical switching devices. By exploiting an effect that is harmful to other SNOM-type measurements, T-SNOM avoids some of the known uncertainties, while operating with relatively high speed and with good signal-to-noise-ratio. Extensive modelling has confirmed the validity of T-SNOM measurements on Fabry-Perot-like microresonators.

\subsection{Far-Field Scattering Microscopy (FSM)}

Although near-field optical measurements, like those described in the preceding section, currently provide the highest available spatial resolution, there are situations where such methods are impractical. This is especially the case if large areas need to be imaged, and/or if the time required for collecting the image data by serial scanning becomes prohibitive, or if the optical structure has a thick cladding layer which makes the evanescent field inaccessible.

An alternative method for looking inside an optical device is using the light that is scattered from the waveguiding regions. A condition for this to work is that small sub-wavelength-size scatter centres should be randomly and homogeneously distributed in the waveguiding and/or cladding materials (Rayleigh scattering regime). In that case, which occurs for many practical optical waveguide technologies, the scattered light intensity is proportional to the guided light intensity. Even in the case of materials showing negligible Rayleigh scattering, the method can sometimes be used if discrete scatterers such as waveguide discontinuities are present at locations of interest in the structure. Then, again the scattered light intensities at such intended discrete scatterers is a measure for the local guided light intensities, so that scatteredlight images can provide information about power ratios at selected locations inside the structure.

Because the scattered light is to be collected by a lens at a distance much larger than the wavelength, the maximum attainable resolution is restricted to the order of a wavelength by the diffraction limit. Although this precludes the imaging of the field structure inside microcavities, such as shown in Fig. 10-10, other useful applications exist, such as waveguide loss measurements [21] and mapping of intracavity power of a microring resonator [22]. In this section we will discuss the application of the method to measure intensity distributions inside a waveguide grating [12].

\subsubsection{Set-Up}

The experimental set-up is schematically shown in Fig. 10-21. Our specific FSM implementation was designed to facilitate the association of scattered light images, that are captured by a CCD camera, with simultaneously measured transmission spectra using a tuneable laser and a photodetector.

Requirements for the camera are linearity and high sensitivity in the wavelength region of interest. In our case the camera could resolve $320 \times 240$ pixels with 12 bits per pixel. In combination with a microscope lens, at the highest magnification each 
Fig. 10-21 Principle of farfield scattering microscopy (FSM), allowing to associate scattered-light images with specific features in device transmission spectra. [12]

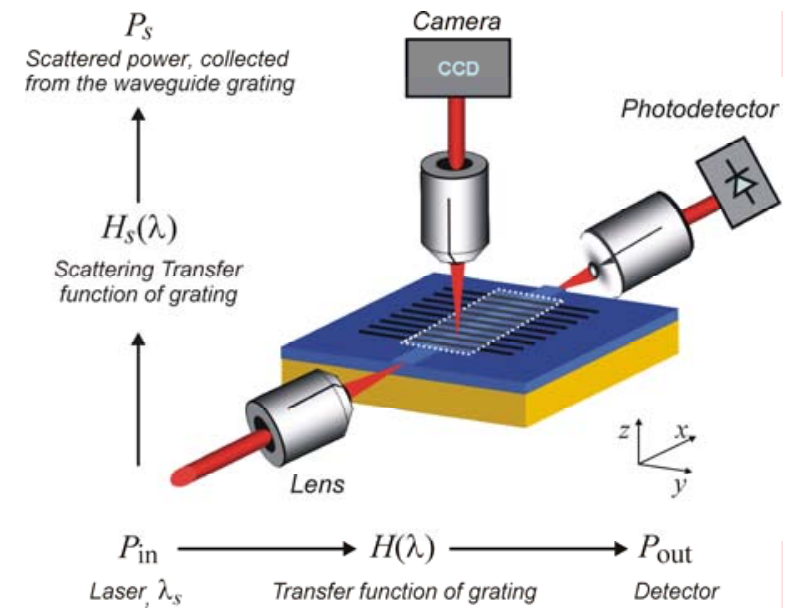

pixel maps to a $800 \mathrm{~nm} \times 800 \mathrm{~nm}$ area on the object under test. The laser could be tuned in the range of $1470-1600 \mathrm{~nm}$ with $1 \mathrm{pm}$ wavelength resolution.

The device under investigation is a waveguide grating such as drawn schematically in Fig. 10-22. This structure can be considered as a Fabry-Perot-like resonator. In the non-grated waveguide sections the usual guided modes can propagate. In the grating section the eigenmodes are the Floquet-Bloch modes of the periodic structure. The transition between grated and ungrated sections are optical discontinuities which partially reflect the guided modes. Hence, a finite-length grating acts as a Fabry-Perot resonator for the Floquet-Bloch modes.

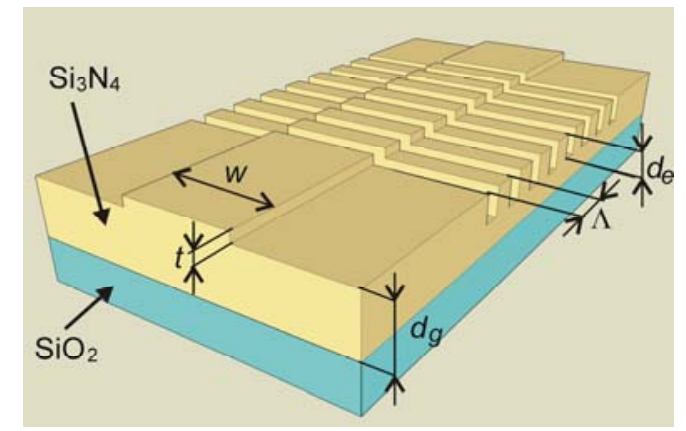

Fig. 10-22 Schematic drawing of shallow ridge waveguide into which a grating has been etched. Materials: $\mathrm{Si}_{3} \mathrm{~N}_{4}$ guiding layer, $n=1.981 ; \mathrm{SiO}_{2}$ bottom cladding, $n=1.445$; top cladding either air $(n=1)$ or polymer $(n=1.5)$. Dimensions: $w=7 \mu \mathrm{m} ; d_{g}=275 \mathrm{~nm} ; t=5 \mathrm{~nm} ; \Lambda=470 \mathrm{~nm} ; d_{e}=50$ $\mathrm{nm}$; device lengths of 500, 1000, and 2000 periods have been used. 


\subsubsection{Measurements}

A typical set of measurement data is shown in Fig. 10-23. The camera images show an envelope of the intensity distribution; the high spatial frequencies of the Floquet-Bloch modes cannot be resolved with this method.

\subsubsection{Interpretation}

The large scale envelope variations (orders of magnitude larger than the wavelength of the light) may be somewhat surprising. This phenomenon can be explained from long-range phase relationships between the Fourier components of the (propagating) Floquet-Bloch mode on the one hand and the overall propagation phase of the field, using a coupled-mode model. These features are reproduced by model calculations using a two-dimensional bidirectional eigenmode propagation method, shown in Fig. 10-24 [12, 13].

As described in [12], the amplitude enhancement in the resonance peak can be used for calculating the group index of slow light that occurs in the regime close to the photonic band edge. For the structure considered here, a maximum group index of 14 was found using this method. Among the several slow-light characterization methods discussed in [12], this method was found to be among the more reliable ones. Related to this also $Q$ of such resonances could be conveniently determined.

In conclusion, the facility to simultaneously obtain both spectral transmission data and spatial scattered light distributions from a finite-length periodic structure
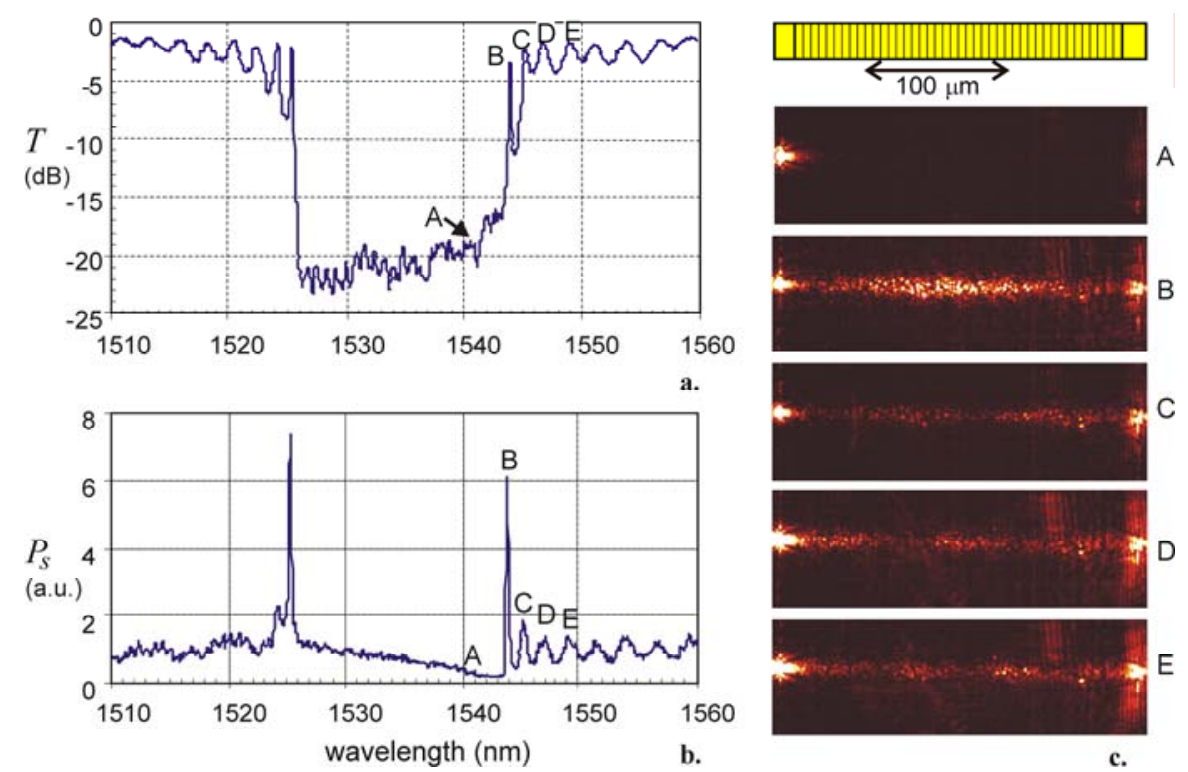

b.

c.

Fig. 10-23 Combined spectral and far-field scattering measurements. a) Normalised transmission spectrum of the waveguide grating, showing a stop-band. b) Spatially averaged scatter spectrum, derived from camera images. c) Typical scattered-light images at 5 different wavelengths, indicated by letters A-E, also shown in graphs a) and $\mathbf{b}) A$ : inside the stopband, $B$ - $E$ Fabry-Perot like grating resonances of order 1-4. Top figure symbolically shows the grating location and a length scale. 

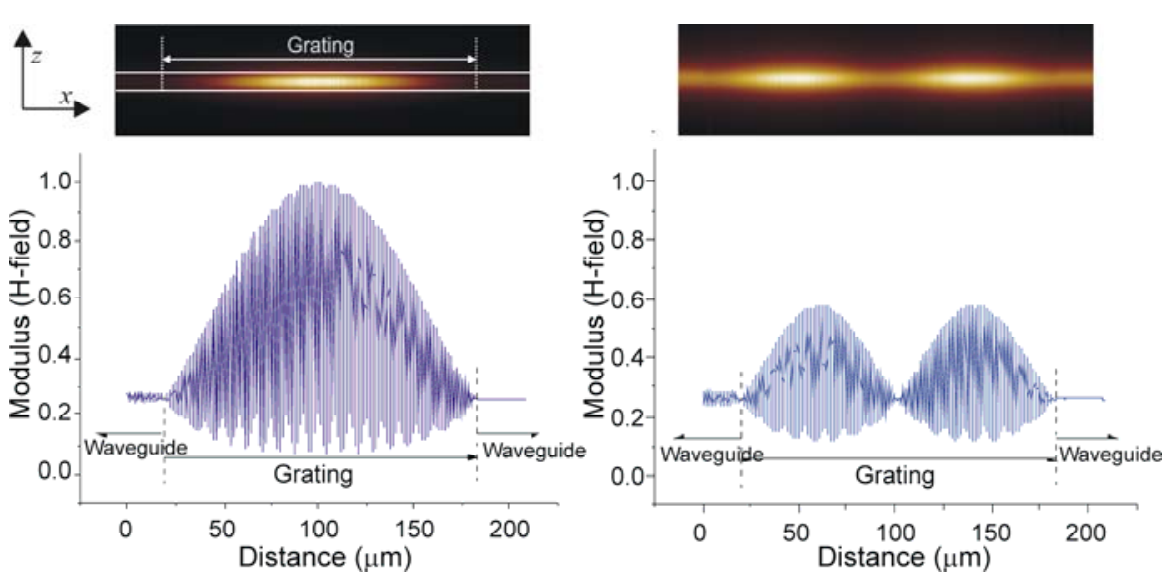

Fig. 10-24 Calculated magnetic field amplitude distribution along the length of the grating. Top field distribution in the grated waveguide cross-section (see coordinate axes in Fig. 10-21). Bottom: field distribution along the centre line of the waveguiding layer. Left lowest order mode (corresponding to label $B$ in Fig. 10-23); right second order mode (label $C$ ). It can be seen that the largescale distribution is actually an envelope of the Floquet-Bloch mode amplitude.

was found helpful for understanding the phenomena near the band edge in such structures. The long-range periodic intensity variation of Fabry-Perot like resonances of the Floquet-Bloch modes that were theoretically predicted could be observed with the far-field scattering microscopy method.

\subsection{Summary}

Three classes of characterization techniques for optical microresonators have been described, viz. analysis of input-output characteristics, transmission-based SNOM, and far-field scattering microscopy. Their typical application fields and specific advantages and disadvantages have been discussed.

Acknowledgments The contributions of Anton Hollink, Kees van der Werf, Hugo Hoekstra, Ronald Dekker, Didit Yudistira, Remco Stoffer, Henk van Wolferen, Marco Gnan, Chris Roeloffzen, Wim Bogaerts, Vinod Subramaniam, Frans Segerink are gratefully acknowledged. This work was supported by NanoNed, a national nanotechnology programme coordinated by the Dutch ministry of Economic Affairs, and was also supported by the European Network of Excellence ePIXnet.

\section{References}

[1] Morand A., Zhang Y., Martin B., Phan Huy K., Amans D., Benech P., Verbert J., Hadji E., Fédéli J.-M.: Ultra-compact microdisk resonator filters on SOI substrate. Opt. Express 14, 12814-12821 (2006) 
[2] Klein E.J., Geuzebroek D.H., Kelderman H., Sengo G., Baker N., Driessen A.: Reconfigurable optical add-drop multiplexer using microring resonators. IEEE Photon. Technol. Lett. 17, 2358-2360 (2005)

[3] Hendrickson J., Richards B.C., Sweet J., Mosor S., Christenson C., Lam D., Khitrova G., Gibbs H.M., Yoshie T., Scherer A., Shchekin O.B., Deppe D.G.: Quantum dot photoniccrystal-slab nanocavities: Quality factors and lasing. Phys. Rev. B. 72 193303(1-4) (2005).

[4] Notomi M., Shinya A., Mitsugi S., Kira G., Kuramochi E.,Tanabe T.: Optical bistable switching action of Si high-Q photonic crystal nanocavities. Opt. Express 13, 2678-2687 (2005)

[5] Bogaerts W., Baets R. Dumon P., Wiaux V., Beckx S., Taillaert D., Luyssaert B., Van Campenhout J., Bienstman P., Van Thourhout D.: Nanophotonic waveguides in silicon-oninsulator fabricated with CMOS technology. J. Lightwave Technol. 23, 401-412 (2005)

[6] Niehusmann J. Vörckel A. Bolivar P.H., Wahlbrink T., Henschel W., Kurz H.: Ultrahighquality-factor silicon-on-insulator microring resonator. Opt. Lett. 29, 2861-2863 (2004)

[7] Foresi J.S., Villeneuve P.R., Ferrera J., Thoen E.R., Steinmeyer G., Fan S., Joannopoulos J.D., Kimerling L.C., Smith H.I., Ippen E.P.: Photonic-bandgap microcavities in optical waveguides. Nature 390, 143-145 (1997)

[8] Notomi M., Shinya A., Mitsugi S., Kuramochi E., Ryu H.Y.: Waveguides, resonators and their coupled elements in photonic crystal slabs: Opt. Express 12, 1551-1561 (2004)

[9] Akahane Y., Asano T., Song B.S., Noda S.: Fine-tuned high-Q photonic-crystal nanocavity. Opt. Express 13, 1202-1214 (2005)

[10] Sauvan C., Lecamp G., Lalanne P., Hugonin J.P.: Modal-reflectivity enhancement by geometry tuning in photonic crystal microcavities. Opt. Express 13, 245-255 (2005)

[11] Little B.E., Chu S.T., Pan W., Kokubun Y.: Microring resonator arrays for VLSI photonics. IEEE Photon. Technol. Lett. 12, 323-325 (2000)

[12] Hopman W.C.L., Hoekstra H.J.W.M., Dekker R., Zhuang L., de Ridder R.M.: Far-field scattering microscopy applied to analysis of slow light, power enhancement, and delay times in uniform Bragg waveguide gratings. Opt. Express 15, 1851-1870 (2007)

[13] Hoekstra H.J.W.M., Hopman W.C.L., Kautz J., Dekker R., de Ridder R.M.: A simple coupled mode model for near band-edge phenomena in grated waveguides. Opt. Quantum Electron. 38, 799-813 (2006)

[14] Hopman W.C.L., Van Der Werf K.O., Hollink A.J.F., Bogaerts W., Subramaniam V., de Ridder R.M.: Nano-mechanical tuning and imaging of a photonic crystal micro-cavity resonance. Opt. Express 14 8745-8752 (2006)

[15] Betzig E., Trautman J.K., Harris T.D., Weiner J.S., Kostelak R.L.: Breaking the diffraction barrier: optical microscopy on a nanometric scale. Science 251, 1468-1470 (1991)

[16] Hsu J.W.P.: Near-field scanning optical microscopy studies of electronic and photonic materials and devices. Mater. Sci. Eng. Rep. 33 1-50 (2001)

[17] Gersen H., Karle T.J., Engelen R.J.P., Bogaerts W., Korterik J.P., van Hulst N.F., Krauss T.F., Kuipers L.: Real-space observation of ultraslow light in photonic crystal waveguides. Phys. Rev. Lett. 94, 073903(1-4) (2005)

[18] Hopman W.C.L., Stoffer R., de Ridder R.M.: High-resolution measurement of resonant wave patterns by perturbing the evanescent field using a nanosized probe in a transmission scanning near-field optical microscopy configuration. J. Lightwave Technol. 25, 1811-1818 (2007)

[19] Hammer M.: Quadridirectional eigenmode expansion scheme for 2D modeling of wave propagation in integrated optics. Opt. Commun. 235, 285-303 (2004)

[20] Hopman W.C.L., Van Der Werf K.O., Hollink A.J.F., Bogaerts W., Subramaniam V., de Ridder R.M.: Experimental verification of a simple transmission model for predicting the interaction of an AFM-probe with a photonic crystal micro-cavity in tapping mode operation. IEEE Photon. Technol. Lett. 20, 57-59 (2008)

[21] McNab S.J., Moll N., Vlasov Y.A.: Ultra-low loss photonic integrated circuit with membranetype photonic crystal waveguides. Opt. Express 11, $2927-2939$ (2003)

[22] Klunder D.J.W., Tan F.S., Van Der Veen T., Bulthuis H.F., Sengo G., Docter B., Hoekstra H.J.W.M., Driessen A: Experimental and numerical study of SiON microresonators with air and polymer cladding. J. Lightwave Technol. 21, 1099-1110 (2003) 